\title{
A Biblioteca do Centro de Estudos do Hospital Felício Rocho: um olhar para a atuação do bibliotecário clínico
}

\author{
Centro de Estudos do Hospital Felício Rocho Library: a look at the performance of the clinical \\ Librarian
}

\author{
Amanda Damasceno de Souza \\ Doutoranda em Gestão de Organização do Conhecimento \\ Universidade Federal de Minas Gerais \\ amandasd81@gmail.com
}

\begin{abstract}
Resumo
O presente artigo discute a atuação do Bibliotecário no âmbito hospitalar onde este profissional é chamado de Bibliotecário Clínico. A Biblioteca Hospitalar desenvolve um trabalho importante no ensino da residência e especialização médica e no desenvolvimento de pesquisa clínica. O objetivo do estudo é relatar a experiência da Biblioteca Hospitalar e do Bibliotecário Clínico no Hospital Felício Rocho, no suporte à equipe médica na busca por informação em saúde. A metodologia foi baseada em relato histórico da biblioteca. Nos resultados foram descritos os usuários, o desenvolvimento da coleção, o acervo e os serviços oferecidos pela biblioteca. As considerações finais reafirmam a importância do Bibliotecário Clínico, as habilidades necessárias para atuar nesse contexto e a necessidade de ampliação dessa experiência para outros hospitais.
\end{abstract}

\section{Palavras-Chave}

Bibliotecas médicas. Gestão de Bibliotecas. Bibliotecário Clínico. Medicina Baseada em Evidência.

\begin{abstract}
This article discusses the role of the librarian in the hospital setting, where this professional is usually named as Clinical Librarian. The hospital library has developed an important work in the medical residency program and clinical research. The objective of this study is to describe the Hospital Library and Clinical Librarian's practices at the Felício Rocho Hospital and how it has helped the medical team in the search for health information. The methodology was based on a historical account of the library. The results described the users, the development of the collection, the collection itself and the services offered by the library. Final considerations reaffirm the importance of the Clinical Librarian, the skills needed to act in this context and the necessity to expand this experience to other hospitals.
\end{abstract}

\section{Keywords}

Medical libraries. Library management. Clinical Medical Librarian. Evidence Based Medicine.

\section{INTRODUÇÃO}

No Brasil, o exercício da profissão de Bibliotecário é regulamentado pela Lei no 4.084, de 30 de junho de 1962, que dispõe:

Art. $1^{\circ}$ A designação profissional de Bibliotecário, a que se refere o quadro das profissões liberais, grupo 19, anexo ao Decreto-lei no 5.452, de 10 de maio de 1943 
(Consolidação das Leis do Trabalho), é privativa dos bacharéis em Biblioteconomia, de conformidade com as leis em vigor. (BRASIL, 1962).

A referida Lei é um marco importante não só para regulamentar o exercício da profissão de Bibliotecário no Brasil, mas, sobretudo, para reconhecer a relevância social de suas atividades. Além do mais, a Lei se notabiliza por descrever as atribuições privativas aos Bacharéis em Biblioteconomia e delimitar as atribuições dos conselhos de fiscalização do exercício da profissão (CUNHA, 1978).

Portanto, para administração, organização, direção e execução de serviços técnicos em bibliotecas, é necessário o diploma de bacharelado em Biblioteconomia, assim como o registro no Conselho Regional de Biblioteconomia (CRB). O art. 2o determina que para o exercício da profissão de bibliotecário somente será permitido a:

a) Bacharéis em Biblioteconomia, portadores de diplomas expedidos por Escolas de Biblioteconomia de nível superior, oficiais, equiparadas, ou oficialmente reconhecidas;

b) Bibliotecários portadores de diplomas de instituições estrangeiras que apresentem os seus diplomas revalidados no Brasil, de acordo com a legislação vigente (BRASIL, 1962).

É interessante observar que as atribuições privativas dos Bacharéis em Biblioteconomia possibilitam a atuação desses profissionais em diversos contextos. Em outras palavras, a Lei no 4.084/1962 não limita a atuação dos bibliotecários, que, no Brasil, tem se expandido cada vez mais num mercado amplo e diversificado. Pelo contrário, a formação acadêmica e profissional dos Bibliotecários possibilita, inclusive, a atuação em áreas e instituições especializadas.

A área de Ciências da Saúde historicamente tem congregado bibliotecários, inclusive em instituições especializadas, como hospitais. O Bibliotecário especialista em saúde pode ser definido como aquele que atua na área médica, na busca por informações específicas para o cuidado ao paciente (WINNING; BEVERLEY, 2003). Isto se deve, segundo Beraquet e Ciol (2010), ao fato de que os médicos têm necessidade de buscar estudos que se assemeIham aos casos clínicos para auxiliá-los na tomada de decisão. Galvão (2008) relata que o profissional da informação em saúde, que atua na organização e recuperação da informação, pode ser nomeado como Bibliotecário Médico, Bibliotecário Clínico ou Informacionista. O Bibliotecário Clínico é o termo utilizado para o profissional que atua junto a equipe médica no fornecimento de informação para o cuidado ao paciente (GALVÃO, 2008). Neste artigo, o termo adotado foi Bibliotecário Clínico, por contextualizar a experiência de atuação desse profissional, no âmbito hospitalar, junto à equipe médica.

O objetivo deste artigo é relatar a experiência do Bibliotecário Clínico no Hospital Felício Rocho (HFR), instituição mantida pela Fundação Felice Rosso, com o intuito de demonstrar a importância da Biblioteca Hospitalar. O artigo relata a história da criação do Centro de Estudos do Hospital Felício Rocho (CEHFR), sua inserção no Núcleo de Ciências da Saúde Felício Rocho (NCSFR) e as atividades recorrentes a uma Biblioteca Hospitalar, e do Bibliotecário em áreas especializadas, como a Medicina.

\section{METODOLOGIA}

A metodologia utilizada foi a descrição histórica da Fundação Felice Rosso, mantenedora do HFR, localizado em Belo Horizonte, Minas Gerais; notadamente no que diz respeito às atividades do Centro de Estudos do HFR. Para tanto, faz-se necessário recuperar também 
como se deu o surgimento do Bibliotecário Clínico nos Estados Unidos da América (EUA) e sua influência nos serviços de informação que são ofertados no HFR.

\subsection{A Fundação Felice Rosso}

A Fundação Felice Rosso foi criada pelo imigrante italiano Nicola Felice Rosso, que chegou ao Brasil em 1882, aos 12 anos de idade. Identificado como carpinteiro na folha de passageiros do navio francês Navarre, exerceu essa atividade no Rio de Janeiro, Petrópolis e Juiz de Fora, onde teve o seu nome abrasileirado para Felício Rocho (RESENDE; MARTINS; GOUVEIA, 2012).

Em 1900, reconhecido como hábil fabricante de esquifes ${ }^{1}$, foi para Belo Horizonte a convite do então prefeito Bernardo Monteiro, para participar da organização dos serviços funerários da Santa Casa de Misericórdia. Com o passar do tempo, interessou-se por novos negócios e ingressou no ramo hoteleiro, tendo adquirido três hotéis e conquistando vultosa fortuna. Por volta de 1921, conheceu o jovem e inteligente advogado Américo Gasparini. Tornaram-se amigos (RESENDE; MARTINS; GOUVEIA, 2012).

Em 1937, sentindo-se adoentado, preocupou-se com o destino de seu patrimônio, uma vez que era solteiro e não tinha herdeiros necessários. Manifestando desejo de doar parte de sua fortuna para um hospital beneficente, foi aconselhado por Américo Gasparini a criar uma fundação hospitalar (RESENDE; MARTINS; GOUVEIA, 2012).

Em 24 de março de 1937, foi lavrada em cartório a escritura de doação da Fundação Ítalo-Brasileira Felice Rosso, com a finalidade de "Criar serviços hospitalares para cuidar dos necessitados e desamparados.". Foi, então, planejada a construção de um Hospital. Felício Rocho participou do lançamento da pedra fundamental, em março de 1937. Faleceu em setembro daquele ano, sem ver seu sonho concretizado (RESENDE; MARTINS; GOUVEIA, 2012).

\subsection{O Hospital Felício Rocho}

Do lançamento da pedra fundamental do Hospital até sua inauguração, em 21 de junho de 1952, passaram-se 15 anos de preocupações, esforços e tenacidade de Américo Gasparini e outros amigos de Felício Rocho, que se propuseram a realizar seu desejo expresso na escritura de doação. Desde os primeiros dias, o Hospital contou com um corpo clínico formado pelos melhores e reconhecidamente mais competentes profissionais da cidade. Tornou-se referência nacional em várias especialidades da Medicina (RESENDE; MARTINS; GOUVEIA, 2012).

As 12 especialidades oferecidas em 1952 transformaram-se em 45 Clínicas, Serviços e Especialidades. Hoje, o HFR está pronto para atender a quaisquer emergências clínicas e cirúrgicas de alta complexidade.

\subsection{O Centro de Estudos do Hospital Felício Rocho}

Os médicos que formaram o primeiro corpo clínico do Hospital costumavam se reunir para trocar informações, ideias, enfim, experiências e conhecimentos. Reuniam-se, informalmente, para discutir casos clínicos e debater artigos de revistas científicas internacionais. O tempo mostrou a importâncias desses encontros, sobretudo para a criação do Centro de Estudos.

\footnotetext{
1 “Caixão mortuário; caixa de madeira usada para enterrar defuntos”. (ESQUIFES, 2020).
} 
Assim, em 1975, aquele grupo propôs a criação de um Centro de Estudos voltado para o incremento do ensino na área da saúde e, com isso, contribuir para o desenvolvimento do corpo clínico, dos residentes e daqueles que cursavam especialização na Instituição. Foi nesse contexto que se originou a criação de uma biblioteca especializada em saúde no HFR (RESENDE; MARTINS; GOUVEIA, 2012).

Embora vinculado tecnicamente ao HFR, o CEHFR é autônomo em termos administrativos. Isto é, o CEHFR é "Uma associação, de fins não econômicos, de caráter científico, cultural, ético e social é de duração indeterminada, com sede e foro em Belo Horizonte." (CENTRO DE ESTUDOS DO HOSPITAL FELÍCIO ROCHO, 2005, p. 1). Conforme os artigos 4ํㅜ e 5o de seu estatuto:

Art. 4ㅇ Podem se inscrever como associados todos os médicos integrantes do corpo clínico e os funcionários de nível universitário que atuam na área de saúde do Hospital Felício Rocho, sendo ilimitado o número de associados, respeitada a condição acima;

Art. 5 Os associados serão divididos nas categorias:

- Associados Fundadores: São os associados que assinaram a ata de fundação do Centro Estudos do Hospital Felício Rocho;

- Associados Efetivos: constituídos pelos membros do corpo clínico e funcionários de nível universitário que atuam no Hospital Felício Rocho na área de saúde;

- Associados Correspondentes: médicos em pleno direito de exercício profissional que não pertencem ao corpo clínico do Hospital Felício Rocho e que tenham sua admissão aprovada pela Diretoria do Centro de Estudos;

- Associados Beneméritos: personalidades nacionais ou estrangeiras que tenham prestado relevantes serviços ao Centro de Estudos do Hospital Felício Rocho (CENTRO DE ESTUDOS DO HOSPITAL FELÍCIO ROCHO, 2005, p. 2-3).

No CEHFR, em consonância com a Lei no 4.084, de 30 de junho de 1962, a presença do Bibliotecário remete a sua fundação. A biblioteca foi criada em meio à ascensão das Teorias sobre a Recuperação da Informação na década de setenta, em que Ciência da Informação inicia as pesquisas com a abordagem de recuperação da informação centrada no usuário (ou humano) (SARACEVIC, 1999). A recuperação da informação médica ganhou destaque nesse período e o Bibliotecário Clínico do CEHFR teve um papel importante na busca pelas melhores evidências disponíveis na literatura para subsidiar a tomada de decisão do corpo clínico, assim como para fomentar e desenvolver a produção científica na Instituição. Em novembro de 2015, o CEHFR foi transferido para o 60 andar e passou a integrar a Unidade de Estudos do NCSFR, reforçando o processo de inovação do HFR.

\subsection{O Núcleo de Ciências da Saúde Felício Rocho}

O crescimento das atividades de ensino e pesquisa desenvolvidas pelos médicos do Hospital, especialmente aquelas ligadas aos cursos de pós-graduação lato sensu e ao incentivo à pesquisa clínica, levaram à criação, em 2003, do Instituto Felício Rocho de Educação Continuada (IFERPEC), também mantido pela Fundação Felice Rosso.

Em 2015, diante da diversidade e do volume das atividades institucionais de ensino, estudo e pesquisa desenvolvidas pelos médicos cada vez mais qualificados, o Conselho Diretor da Fundação decidiu transformar o IFERPEC em uma unidade que melhor congregasse as atuais atividades de ensino, estudo e pesquisa. Assim, em novembro do mesmo ano foi 
inaugurado o NCSFR, composto por 1. Unidade de Ensino 2. Unidades de Pesquisa 3. Unidade de Estudos.

A Unidade de Ensino tem a atribuição de planejar, coordenar, supervisionar, auxiliar e avaliar os programas de Residência e Especialização Médicas. Além de coordenar e organizar as atividades da Educação Médica Continuada e garantir a relação da residência respeitando as regras e exigências do Ministério da Educação (MEC), assim como acompanhar o desenvolvimento técnico dos mesmos.

A Unidade de Pesquisa tem por finalidade promover o desenvolvimento e a integração da pesquisa clínica na Fundação Felice Rosso e no HFR. Desempenha a função de facilitadora das pesquisas clínicas realizadas na instituição e estabelece diretrizes e políticas relativas à realização dessas.

A Unidade de Estudos é composta pelo Centro de Estudos (CEHFR) e por uma biblioteca. O CEHFR apresenta Comprovante de Inscrição e de Situação Cadastral de Pessoa Jurídica (CNPJ) próprio, é mantido pelo corpo clínico do HFR, além de regido por Estatuto individual. São atribuições do CEHFR: manter a biblioteca especializada em saúde e contribuir para o financiamento de eventos técnico-científicos de interesse das clínicas do Hospital Felício Rocho. A biblioteca da Unidade de Estudos do NCSFR, mantida pelo CEHFR, caracteriza-se como biblioteca especializada em saúde (NÚCLEO DE CIÊNCIAS DA SAÚDE FELÍCIO ROCHO, 2019).

\section{O BIBLIOTECÁRIO CLÍNICO}

O Bibliotecário que atua na área de saúde foi chamado na década de 1970 pela bibliotecária americana Gertrude Lamb de Clinical Medical Librarian (CML). Em português, Bibliotecário Clínico. Esse profissional desempenha um papel estratégico ao identificar as necessidades informacionais de médicos, docentes e residentes garantindo-lhes o acesso às principais evidências publicadas (VAN KESSEL, 2012). Lamb, em 1971, criou na Escola de Medicina da Universidade do Missouri-Kansas City (UMKC), o primeiro programa de CML para apoiar a informação hospitalar. Lamb, ainda em 1971, nos EUA, identificou uma lacuna entre o que a Medicina como disciplina conhecia sobre boas práticas de cuidado ao paciente e o conhecimento que era realmente aplicado ao cuidado desse paciente. Nesse contexto, percebeu-se uma oportunidade para bibliotecários ajudarem a realizar essa conexão entre a literatura médica e o cuidado do paciente. A Bibliotecária Lamb foi pioneira no conceito de bibliotecários que participam de rondas clínicas para identificar necessidades de informação, relacionadas a casos clínicos (ARCARI; LAMB, 1977; HARRISON; BERAQUET, 2010).

No primeiro programa de CML da UMKC, o Bibliotecário Clínico era designado para acompanhar o processo de internação e a ronda dos leitos junto à equipe de saúde. A finalidade, então, era prover informações necessárias ao atendimento do paciente. O Bibliotecário Clínico tinha o papel fundamental de garantir que os médicos tivessem acesso à literatura mais atual e às melhores informações disponíveis. Sobre a atuação do Bibliotecário Clínico, Hill $(2008$, p.17) afirma que esse profissional precisa "[...] fornecer informação de qualidade aos profissionais de saúde no momento de necessidade de apoiar a tomada de decisão clínica.".

Lipscomb (2000) relata que o Bibliotecário Clínico pesquisava literatura médica atual para responder ao cuidado com o paciente, e prover os médicos com artigos relevantes. Os programas de CML foram se adaptando às necessidades de cada instituição, em decorrência disso, houve a ampliação dos programas e aderência de vários hospitais de 1971 a 1977, nos 
EUA. Os programas de CML também se desenvolveram devido aos seguintes acontecimentos:

- 1974 - Journal of the American Medical Association anuncia o suporte aos Clinical Librarians.

- 1981 - Criação da Medical Library Association (MLA).

- 1982 - Criação da revista Clinical Librarian Quarterly, publicada até 1986.

O conceito de Bibliotecário Clínico é tido como uma inovação no âmbito das bibliotecas hospitalares, sendo reforçado pelo movimento da Medicina Baseada em Evidência (MBE). O Bibliotecário Clínico agrega mais versatilidade à Biblioteconomia, e demonstra ser um mercado em potencial devido à sua atuação junto a equipe médica na busca pela evidência científica (PIRES; RIBEIRO; KLEBERSSON, 2013). Além disso, esse profissional possibilita a gestão de conhecimento nos hospitais, ao atuar junto à equipe médica, no auxílio ao desenvolvimento de produção científica e pesquisa clínica (BERAQUET; CIOL, 2009).

No início da década de 1990, um grupo de pesquisadores da Universidade McMaster, no Canadá, visando ao ensino e uso de evidências científicas aliado à prática médica, inicia um movimento denominado MBE (FERNANDES, 2015). A MBE é definida por Sackett et al. (1996) como:

[...] o uso consciente, explícito e criterioso das melhores evidências atuais disponíveis na tomada de decisões sobre o atendimento aos pacientes de forma individual. A prática da medicina baseada em evidência significa integrar conhecimentos clínicos individuais com a melhor evidência clínica externa disponível proveniente de pesquisas sistemáticas de informações (SACKETT et al., 1996, p. 71). ${ }^{2}$

Lopes (2000) ressalta que, na prática da MBE, a experiência clínica é integrada com a capacidade de analisar criticamente a informação científica, com a finalidade de melhorar a assistência médica prestada ao paciente. No contexto da MBE, o Bibliotecário Clínico atua na elaboração, orientação, redação, submissão de artigos científicos e projetos de pesquisa acadêmica e clínica, na construção de repositórios e biblioteca digitais e na capacitação em bases de dados. O Bibliotecário Clínico possibilita o acesso à informação (SILVA, 2005), além de contribuir para melhorar a representação do conhecimento em Sistemas de Informação em Saúde (SIS) (BERAQUET; CIOL, 2010).

O Bibliotecário Clínico tem uma atuação fundamental em bibliotecas hospitalares, ao auxiliar a equipe médica na pesquisa científica, na compreensão da doença e no acompanhamento dos avanços dos tratamentos (HENDERSON, 2016). Além disso, Gerber (2017) aborda que o bibliotecário desempenha um papel estratégico na Educação Médica Continuada ao ensinar métodos de pesquisa, orientar a elaboração de projetos de pesquisa, conduzir pesquisa usando bancos de dados como PubMed ${ }^{\circledR}$ e outros recursos. No âmbito hospitalar, a Biblioteconomia Clínica cumpri a missão de:

[...] tornar a informação relevante e disponível no momento em que é solicitada. No hospital, o bibliotecário clínico leva a biblioteca ao usuário, antecipa suas ques-

\footnotetext{
${ }^{2}$ Tradução do original: Evidence based medicine is the conscientious, explicit, and judicious use of current best evidence in making decisions about the care of individual patients. The practice of evidence based medicine means integrating individual clinical expertise with the best available external clinical evidence from systematic research.
} 
tões e tenta oferecer a informação adequada até mesmo antes de ser solicitado. (BERAQUET; CIOL, 2010, p.133).

Harrison e Beraquet (2010), em um estudo realizado com Bibliotecário Clínico do Reino Unido, relataram que a confiança e boa relação de trabalho entre profissionais de saúde e bibliotecários são os elementos essenciais para desenvolver serviços de Bibliotecário Clínico, são oferta de serviços com a tempestividade e excelência requisitados no contexto da saúde.

Além do mais, a atuação do Bibliotecário Clínico em hospitais exige habilidades para lidar com a complexidade da informação em saúde. Beraquet e Ciol (2009) citam algumas habilidades necessárias ao bibliotecário que atua na área médica, entre elas:

a) bom relacionamento com a equipe médica;

b) capacidade de elaborar perguntas e aprender sobre questões clínicas e científicas;

c) conhecimentos sobre anatomia e fisiologia;

d) domínio de vocabulário médico;

e) experiência em pesquisa nas bases de dados;

f) conhecimento em medicina baseada em evidência;

g) conhecimento em pesquisa clínica;

h) conhecimento em epidemiologia.

Além dessas habilidades, o Bibliotecário Clínico precisa ser proativo e levar os serviços da Biblioteca Hospitalar ao usuário, ou seja, disponibilizar fontes de informação para o médico, em todo o ambiente hospitalar. Lyon et al. (2015) abordam que o Bibliotecário Clínico, além de ser treinado em disciplinas da área médica e em habilidades especializadas em saúde, ele precisa conhecer a terminologia médica, a cultura clínica, a política e o meio ambiente do hospital em que atua. É um profissional que pode auxiliar na elaboração e formatação de Guidelines e manuais institucionais.

O Bibliotecário Clínico precisa compreender as evidências científicas em saúde e buscar formas inovadoras de prestação de serviços, utilizando as novas tecnologias, as terminologias clínicas e o prontuário do paciente para auxiliar, também, nas pesquisas que utilizam dados biomédicos. Cabe ressaltar que conhecer outros idiomas, como inglês e espanhol, é fundamental para realizar pesquisas em bases de dados internacionais. Ao realizar as pesquisas em bases de dados também é necessário saber avaliar os resultados das buscas, conhecer os principais periódicos da área de Medicina, conhecer protocolos de condutas das especialidades médicas, conhecer os tipos de estudos: revisão sistemática, estudos randomizados, relatos de casos, além de saber avaliar as informações em saúde e assim repassar à equipe em saúde, somente conteúdo de qualidade.

\section{O BIBLIOTECÁRIO CLÍNICO E A TOMADA DE DECISÃO EM SAÚDE}

O Bibliotecário Clínico qualificado para identificar recursos apropriados, com interfaces complexas, pode dar suporte à equipe médica na MBE ao usar seu tempo e expertise para articular uma questão clínica na busca pelas melhores evidências. Isso porque, para a equipe médica falta tempo e experiência na articulação entre a questão clínica e a pesquisa nas bases de dados (CIOL; BERAQUET, 2009; YAEGER; KELLY, 2014). Hageman (2019) afirma que bibliotecários são úteis em fornecer dados clínicos, referências atuais e históricas para o 
melhor atendimento do paciente. Para Hageman (2019) o Bibliotecário Clínico fornece à equipe médica artigos relacionados a MBE. Além disso, o Bibliotecário Clínico pode auxiliar o médico na documentação de projetos clínicos, no gerenciamento de dados e na preparação de revisões sistemáticas. O Bibliotecário Clínico também pesquisa e disponibiliza os recursos necessários para estudantes de medicina, residentes, médicos assistentes, pesquisadores médicos, e outros profissionais de saúde em ambientes hospitalares e acadêmicos (HAGEMAN, 2019).

No âmbito da Biblioteca Hospitalar, o Bibliotecário Clínico é considerado um professor, por instruir o corpo clínico no uso da literatura. Freitas, Gomes e Santos (2017) citam o Bibliotecário Clínico como mediador da informação em saúde. A Biblioteca Hospitalar deve oferecer estrutura física adequada a seus usuários, e serviços para atender à necessidade de informação em saúde na tomada de decisão. Assim a mediação da informação deve ser planejada e pensada no contexto hospitalar, suas demandas por informação em seus suportes físicos e digitais (FREITAS; GOMES; SANTOS, 2017).

No contexto da tomada de decisão em saúde, o Bibliotecário Clínico exerce quatro funções essenciais 1) é eficaz na economia de tempo dos profissionais de saúde, 2) fornece informações relevantes, 3) fornece informações úteis e 4) oferece serviços de alta qualidade (BRETTLE et al., 2011). Por sua experiência na recuperação de informação, o Bibliotecário Clínico encontra os artigos mais relevantes, é capaz de destacar os pontos pertinentes, escrever resumos para as equipes médicas e apontar opiniões divergentes da literatura em saúde (SCHACHER, 2001). Schacher (2001) argumenta que Bibliotecário Clínico fornece muito mais do que pesquisas rápidas, uma vez que a formação e o treinamento deste profissional permitem que ele entenda e antecipe perguntas feitas em rondas clínicas e, consequentemente, analisa na literatura científica quais são as informações necessárias à equipe médica.

\section{A BIBLIOTECA HOSPITALAR DO CENTRO DE ESTUDOS DO HOSPITAL FELÍCIO ROCHO}

Shera (1972) conceitua a biblioteca como um espaço que contribui para o sistema de comunicação total na sociedade, criadas como um instrumento para maximizar a utilidade dos registros documentais para os benefícios sociais, ao trabalhar para o indivíduo e através desse indivíduo, atingir a sociedade.

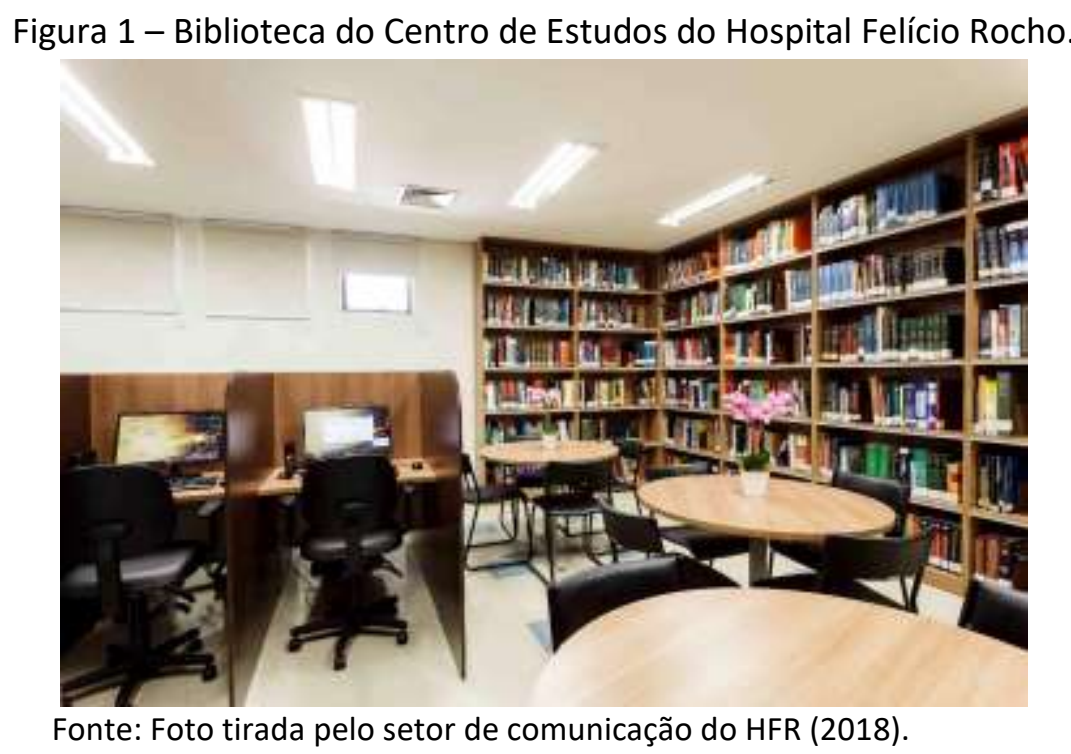


Nesse sentido, a Biblioteca do CEHFR apresenta um espaço moderno com estantes para acervo de livros e periódicos, mesas de estudos e cabines individuais para uso de computador com acesso à Internet. A Biblioteca Hospitalar é o instrumento que busca maximizar a informação em saúde no cuidado ao paciente, e assim, beneficiar a sociedade. A Figura 1 mostra a estrutura atual da Biblioteca do CEHFR localizada no 6음 do Hospital Felício Rocho.

A biblioteca, ao compartilhar seus dispositivos informacionais, concretiza o processo de mediação. É através dessa ação que o usuário tem acesso à informação por meio de seu acervo, estrutura física e de pessoal, de maneira que isso contribua para a construção do conhecimento. A biblioteca enquanto colaboradora para o processo de ensinoaprendizagem, necessita produzir e compartilhar práticas voltadas para o acesso, busca e utilização de seus dispositivos informacionais, visando, sobretudo, as necessidades de informação de seus usuários. Com isso, o bibliotecário deve assumir o papel efetivo de mediador da informação (FREITAS; GOMES; SANTOS, 2017).

As práticas da Biblioteca do CEHFR, os usuários, o desenvolvimento de coleções e os serviços serão descritos a seguir.

\section{a) Usuários da Biblioteca}

A Biblioteca do CEHFR é mantida por seus associados conforme o estatuto. O objetivo primordial é auxiliar com informação focada nas especialidades atendidas no hospital. Assim, seus usuários são aqueles que demandam por informação para tomada de decisão em saúde, na instituição:
a) Corpo Clínico;
b) Residentes;
c) Equipe assistencial: enfermagem, psicologia, fisioterapia, fonoaudiologia, assis- tência social, nutrição.
d) Estagiários de medicina, enfermagem, psicologia, fisioterapia, fonoaudiologia, as- sistência social, nutrição e outros.

Os residentes são os usuários mais frequentes da biblioteca. Acredita-se que isso se justifique em razão do estágio da carreira desses profissionais. Eles estão em constante aprendizado para a formação nas especialidades que atuam, o que torna necessária a realização de produção científica e também o desenvolvimento de uma monografia no final de sua residência ou especialização.

Os usuários da biblioteca do CEHFR necessitam de informação para subsidiar e qualificar a tomada de decisão em saúde, a produção científica, ministrar aulas e conferências, atividade de ensino e de aprendizado, desenvolver novas técnicas cirúrgicas, realizar discussão sobre casos clínicos, elaborar protocolos, até a gestão hospitalar. Embora o acervo da biblioteca contempla somente assuntos relacionados à Medicina, seus serviços atendem também à equipe multiprofissional.

\section{b) Desenvolvimento da coleção}

Em um contexto no qual a disponibilização de recursos de informação requer, cada vez mais, o formato digital, o desenvolvimento de coleções é um tema cuja importância está diretamente ligada à presença da Biblioteca Hospitalar. Vergueiro $(1989$, p. 13) relata que a 
biblioteca precisa disponibilizar fontes de informação aos seus usuários e o bibliotecário torna-se "[...] uma ponte entre o acervo sobre o qual tem a responsabilidade e um usuário que cuja exigência cresce exponencialmente.".

A política de desenvolvimento de coleção da Biblioteca do CEHFR visa atender às necessidades de informação em saúde de seus usuários e estabelecer os critérios de seleção, aquisição e descarte do respectivo acervo. A seleção é realizada pelo Bibliotecário Clínico juntamente com o corpo clínico, os assuntos de interesse se referem às especialidades medicas do Hospital e o descarte é realizado com base na desatualização das obras ao realizar o inventário anual da coleção.

O desenvolvimento de coleções é trabalhado em consonância com os objetivos da biblioteca e da instituição, e busca atingir seus usuários, ou seja, busca atender ao corpo clínico para suporte à $\mathrm{MBE}$, como a formação da residência e da especialização. Na Biblioteca do CEHFR, o desenvolvimento de coleções está em conformidade com as características das especialidades médicas presentes no HFR e contempla as necessidades de informação da residência e da especialização. As decisões sobre seleção e descarte de livros são tomadas em acordo com o coordenador de cada especialidade médica, com base em sugestões de sua equipe e dos residentes. $O$ desenvolvimento de coleções em Biblioteca Hospitalar deve ser realizado de modo a oferecer fontes de MBE, através das quais o médico tenha acesso à informação, não somente na biblioteca, mas em aplicativos móveis e por terminais de computador, em todo o hospital. Assim, as assinaturas de base de dados são realizadas de forma institucional para serem acessadas em toda a instituição.

Atualmente, a aquisição de acervo é realizada por meio de compra e doação de acervo. O CEHFR disponibiliza uma verba para cada especialidade médica da instituição, que poderá escolher entre a aquisição do livro, periódico científico ou base de dados, no formato de acervo físico ou virtual. O desenvolvimento de coleções deve ser contínuo e ter o foco em seu usuário e nas mudanças e necessidades de informações que demonstram ser tão complexas na área de medicina. Para essa tarefa, o Bibliotecário Clínico, ao realizar o desenvolvimento de acervo, precisa conhecer as principais fontes de informações em saúde de cada especialidade médica do hospital em que atua, além de estabelecer uma boa comunicação com a equipe médica para decidir qual acervo atenderá às necessidades de informação.

Entretanto, acervos na área de Medicina são onerosos e se desatualizam rápido. Uma alternativa para suprir demandas específicas de informação é a realização de cooperação bibliográfica. As bibliotecas, devido à impossibilidade de adquirir, de forma isolada, o acervo necessário para atender a seus usuários, utiliza serviços cooperativos em rede eletrônica para suprir demandas de informação (CUNHA, 2018). No Brasil, o programa de colaboração entre bibliotecas é conhecido como Programa de Comutação Bibliográfica (COMUT) do Instituto Brasileiro de Informação em Ciência e Tecnologia (IBICT).

O COMUT atende às demandas específicas de artigos para uma área como a Medicina, que apresenta diversidade de publicações e atualizações constantes, pela emissão de cópias de documentos técnico-científicos, de maneira ágil (IBICT, 2012). A Biblioteca do CEHFR realiza COMUT com a Biblioteca da Faculdade de Medicina da Universidade Federal de Minas Gerais (UFMG).

A Biblioteca do CEHFR caracteriza-se como biblioteca especializada em saúde, sendo seu acervo composto por livros, periódicos, monografias, dissertações, teses e outros materiais em formato físico ou digital (NÚCLEO DE CIÊNCIAS DA SAÚDE FELÍCIO ROCHO, 2019). O acervo da Biblioteca do CEHFR é formado por obras relacionadas às especialidades médicas atendidas no Hospital (HOSPITAL FELÍCIO ROCHO, 2020). 
Na catalogação, é utilizada a classificação da NLM Classification 2019 Summer Edition da National Library of Medicine (NLM), tabela de Cutter e o vocabulário controlado da Bireme Descritores em Ciências da Saúde (DeCS³) (WILLIS, 2019; SANTOS; MOLLICA; GUEDES, 2020).

O desenvolvimento de coleções em saúde é uma tarefa desafiadora. Para suprir as carências e demandas por atualização constante do acervo, é necessário seguir alguns passos:

a) solicitar sugestões dos médicos que publicam e atuam como preceptores, na residência e na especialização para adquirir acervo que atenda ao ensino (o médico é um parceiro da biblioteca no desenvolvimento de coleções);

b) conhecer a excelência da instituição para antever quais assuntos serão demandados;

c) realizar estatísticas de empréstimo de livros para verificar quais obras precisam ser adquiridas em versão atualizada;

d) estabelecer parcerias com outras bibliotecas para as demandas por busca ativa de artigos específicos em periódicos sem assinatura institucional.

Por outro lado, utilizar as novas tecnologias da informação e buscar inovações é um grande diferencial para a Biblioteca Hospitalar. Recursos como bibliotecas digitais e virtuais são essenciais para disponibilizar informações em saúde à equipe médica em tempo hábil.

Em 2017, foi criada a Biblioteca Virtual do CEHFR ${ }^{4}$ com objetivo de ser uma fonte de informação capaz de disponibilizar e possibilitar o compartilhamento do conhecimento produzido no HFR (Figura 2). Para isso, ela agrega o catálogo de livros e, em formato digital, disponibiliza as monografias da residência e da especialização do HFR. Tornou-se assim um recurso para reunir, disponibilizar, preservar e divulgar a produção científica da residência, da especialização e do corpo clínico da instituição. Além disso, a Biblioteca Virtual do CEHFR é uma ferramenta para a gestão do conhecimento, um recurso que os médicos da instituição utilizam como repositório institucional para suas aulas, palestras, seus trabalhos publicados ou apresentados em congressos e na promoção da educação continuada (PRAXIS SOFTWARES GERENCIAIS, 2020) (Figura 3).

Figura 2 - Biblioteca Virtual do Centro de Estudos do Hospital Felício Rocho

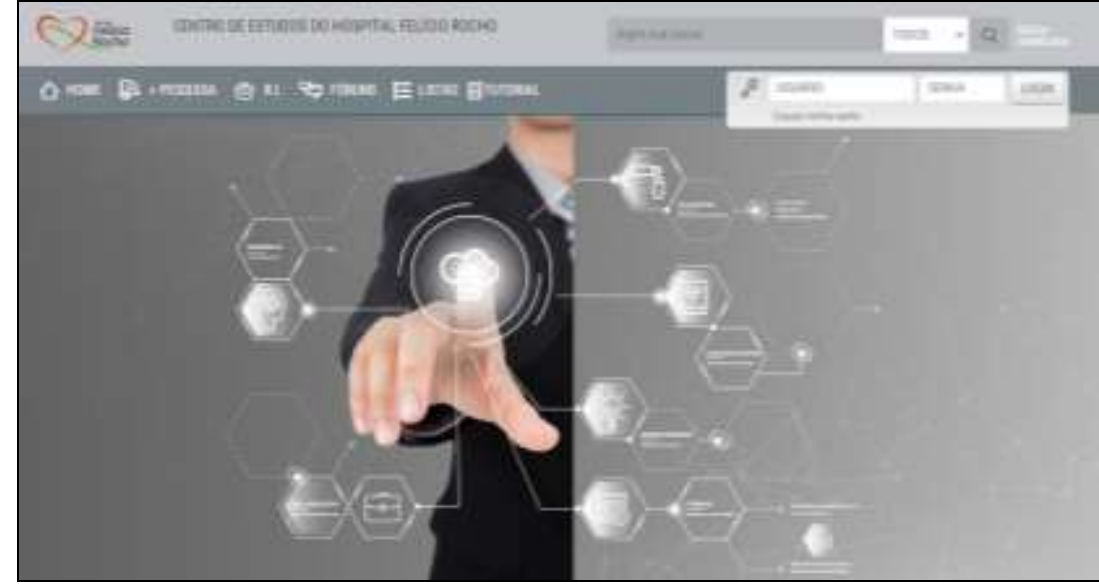

Fonte: CEHFR (2020).

\footnotetext{
${ }^{3}$ Endereço eletrônico: http://decs.bvs.br/

${ }^{4}$ Endereço eletrônico: https://centroestudoshfr.i10bibliotecas.com.br/
} 
O software utilizado na gestão da Biblioteca Virtual é o i10bibliotecas! @ , software brasileiro de gerenciamento de acervos com rede social de leitores. A escolha do i10 bibliotecas! (c para gestão do acervo físico e virtual se deve ao fato de possibilitar a integração da Biblioteca Digital com a funcionalidade de repositório, o que permite o armazenamento de documentos em diversos formatos digitais (áudios, vídeos, fotos, folhetos, folders institucionais) entre outros (PRAXIS SOFTWARES GERENCIAIS, 2020).

Figura 3 - Repositório institucional do Centro de Estudos do Hospital Felício Rocho.

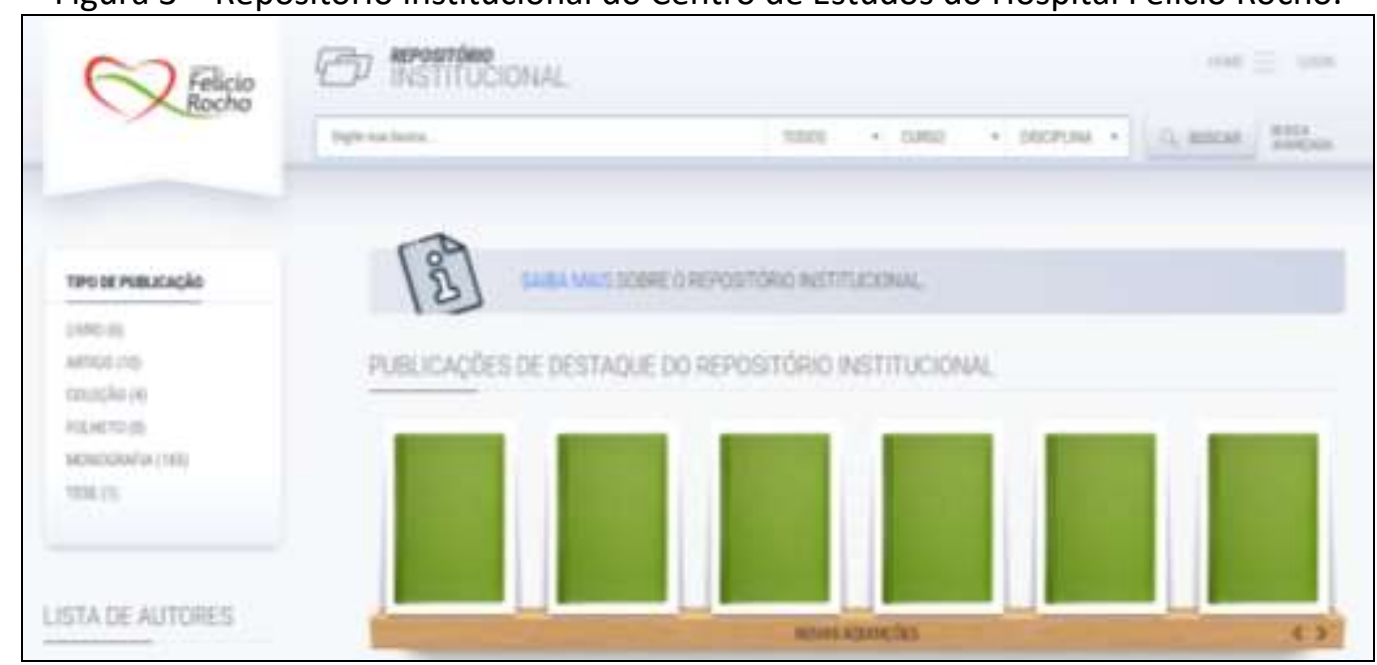

Fonte: CEHFR (2020).

\section{c) Serviços oferecidos}

Como suporte à MBE, a biblioteca hospitalar apresenta uma função primordial ao disponibilizar serviços personalizados para atender às necessidades de informação e de fomento à pesquisa. Para prestar melhor assistência aos pacientes, o corpo clínico demanda por produtos e serviços de informação em saúde. Assim, os serviços do Bibliotecário Clínico beneficiam o atendimento ao paciente, economizam tempo e custos para o médico (BRETTLE et al., 2011). O serviço de referência apresenta a demanda mais representativa da Biblioteca Hospitalar, que faz a mediação entre o conhecimento e o usuário (JESUS; CUNHA, 2012).

Após a mudança da Biblioteca do CEHFR para o NCSFR em 2015, foram agregados novos serviços e realizados estudos de usuários da biblioteca, para auxiliar no desenvolvimento de coleções. Os serviços oferecidos aos usuários realizados pela Biblioteca do CEHFR serão descritos a seguir:

a) empréstimo domiciliar de acervo;

b) acesso à Internet: disponibilização de computadores na biblioteca com acesso liberado a Internet para o corpo clínico, residentes e especializandos;

c) realização de levantamento bibliográfico nas bases de dados e nas fontes de informação em saúde;

d) busca ativa de artigo e envio por e-mail;

e) Disseminação Seletiva da Informação (DSI): envio de sumário corrente sobre novidades, novas aquisições e artigos publicados nas principais revistas médicas; 
f) realização de COMUT: comutação bibliográfica com biblioteca da área médica para obtenção de cópias de documentos técnico-científicos disponíveis em acervos de bibliotecas de todo o Brasil e outros países;

g) reprografia;

h) acesso institucional a sumários de MBE;

i) treinamentos no acesso à base de dados em saúde e em fontes de MBE;

j) elaboração de manuais da biblioteca e do Comitê de Ética em Pesquisa do Hospital Felício Rocho (CEPHFR);

k) orientação de projetos de pesquisa ao Comitê de Ética em Pesquisa (CEP);

l) orientação de projetos de pesquisa e formatação bibliográfica;

m) assessoria de bioestatística no desenho metodológico, cálculo amostral e elaboração de questionário para coleta de dados.

Os serviços realizados pela biblioteca visam atender às necessidades de informação do corpo clínico para assistência ao paciente, promover a produção científica e a pesquisa clínica na instituição. Por meio do serviço de levantamento bibliográfico, a MBE torna-se viável pela realização de revisões sistemáticas em bases de dados da saúde, como o PubMed $^{\circledR}$ (FIORINI; LIPMAN; LU, 2017).

Figura 4 - Formulário padronizado para realização de levantamento bibliográfico

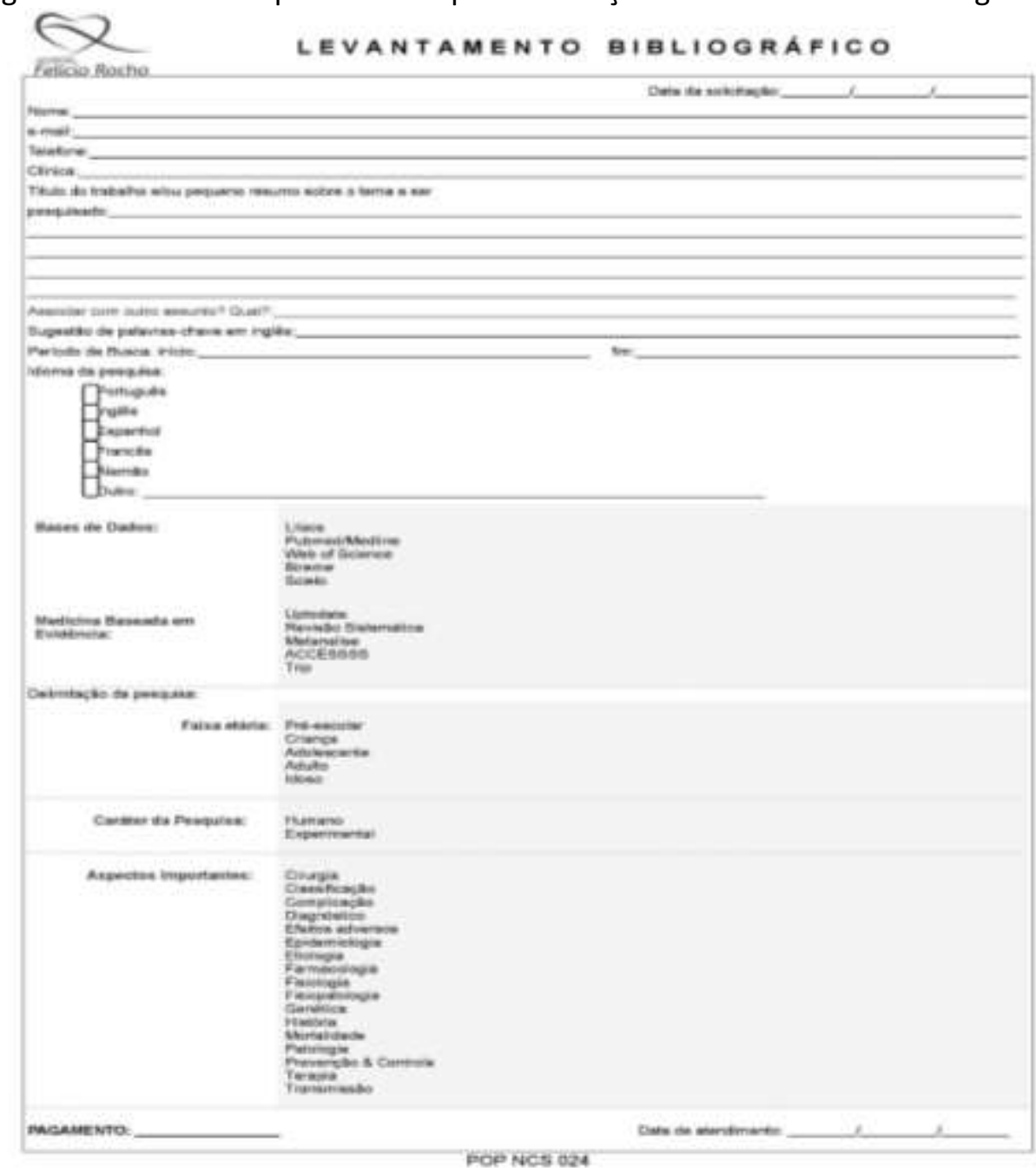

Fonte: Elaborado na Biblioteca do CEHFR e disponível no MV-Qualidade/HFR (2020). 
Os bibliotecários clínicos têm se envolvido com a atividade de realização de revisões sistemáticas desde que esse gênero de publicação surgiu na década de 1990. Algumas das funções em potencial para os bibliotecários nessa atividade se refere à realização da pesquisa, a seleção de fontes, ao gerenciamento de citações, ao fornecimento de documentos e à avaliação crítica dos resultados (SPENCER; ELDREDGE, 2018). Este serviço é oferecido in loco, por telefone e também por e-mail, pelo envio de um formulário padrão que contém os principais aspectos necessários à realização da revisão sistemática (Figura 4).

Ao realizar o serviço de levantamento bibliográfico, é imprescindível utilizar um formulário padronizado para registrar as especificidades da informação solicitada. Neste momento, o bibliotecário, além de preencher os campos pré-definidos nos formulários sobre os aspectos principais da informação requerida, deve dialogar com o usuário e entender o motivo daquela solicitação. O formulário desenvolvido pela Biblioteca do CEHFR contempla um campo para identificação do usuário e a especialidade em que ele atua, um campo para descrever o tema de pesquisa e um campo para os descritores, um campo para definir idioma, um para base de dados, e um campo para os aspectos sobre o paciente e a enfermidade pesquisada.

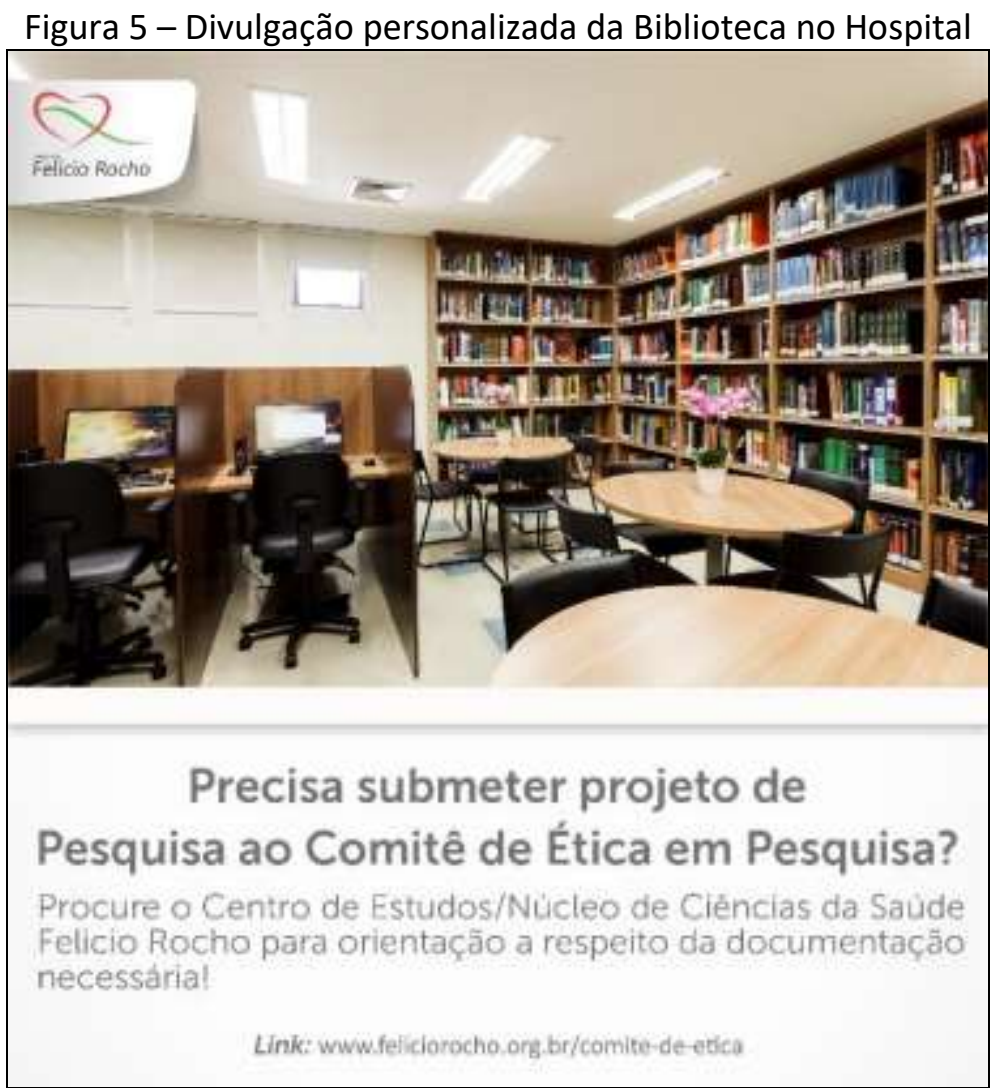

Fonte: Setor de comunicação do HFR (2019).

Para promover a produção científica e a pesquisa clínica são oferecidos pelo Bibliotecário Clínico os serviços de orientação a submissão e elaboração de projetos de pesquisa ao CEP/HFR, além da orientação, sobre a formatação dentro das normas Associação Brasileira de Normas Técnicas (ABNT) e Vancouver (BAHADORAN et al., 2020). Por fim, o Bibliotecário Clínico é responsável pela elaboração de manuais da Biblioteca e do CEP/HFR. A divulgação dos serviços da biblioteca e novas aquisições de acervo é realizada de forma personalizada (Figura 5). 


\section{CONSIDERAÇÕES FINAIS}

A crescente especialização da Saúde, seus serviços e a informação em saúde, se coloca como uma área potencial para atuação do Bibliotecário Clínico. Esse contexto apresenta todos os ingredientes em uma receita para desafios e também para oportunidades de serviços especializados de informação e, assim, coloca uma nova e melhor maneira de beneficiar os usuários das bibliotecas hospitalares. Cabe ao Bibliotecário Clínico torna-se o profissional que, além de propiciar acesso às informações relevantes e metodologicamente adequadas, para a equipe em saúde, possa ir além e atuar, também na produção científica e nas questões ética, relativas aos dados dos pacientes e na mediação da informação em saúde. O Bibliotecário Clínico deve atuar como mediador da informação em saúde para promover o acesso, o uso e a apropriação da informação pela equipe médica, no desenvolvimento de novos conhecimentos e na assistência ao paciente. Para isso, deve analisar a literatura em saúde, visando não somente a sua recuperação, mas também ao desenvolvimento de ações para divulgação de evidências.

O Bibliotecário Clínico integra a equipe clínica multiprofissional de saúde, pelo seu papel de procurar, filtrar e fornecer melhores evidências para decisões clínicas, dando suporte à equipe médica através de levantamentos bibliográficos, normalização de publicações técnico-científicas, organização de arquivos de prontuários, arquivos documentais e de coleções. Para isso, ele deve conhecer a terminologia médica, as bases de dados e periódicos da área, interagir com a equipe médica, saber elaborar perguntas e aprender sobre questões clínicas e científicas, aprender sobre anatomia e fisiologia, estudar a MBE, adquirir noções de pesquisa clínica, de epidemiologia e de bioestatística.

A Biblioteca do CEHFR é um espaço de mediação da informação nos mais diversos suportes, físico e digital, no âmbito hospitalar. Além, disso é também um espaço de estudo, ensino e desenvolvimento de pesquisa hospitalar. Seu diferencial de sucesso e inovação está ligado, principalmente, aos serviços informacionais e de apoio à apreciação ética oferecidos aos usuários.

O Bibliotecário Clínico do HFR tem uma importante função educativa, ao atuar na esfera da construção do conhecimento da residência e especialização médica. É um profissional que necessita da capacitação constante devido às mudanças da Medicina. Com isso, a participação em eventos científicos médicos, como simpósios e congressos, é uma alternativa para a capacitação e atualização sobre demandas de informação do corpo clínico.

Como perspectivas futuras, o Bibliotecário Clínico deve estar atualizado em relação à Tecnologia da Informação, promover em sua biblioteca de atuação o acesso a artigo de texto completo, realizar constantemente treinamentos da equipe médica em bases de dados, e dar ênfase na prática da saúde baseada em evidências. Por fim, tem a missão de divulgar o conceito de Bibliotecário Clínico para outros Hospitais que ainda não possuem biblioteca.

Com a descrição deste relato de experiência, espera-se que o exemplo do HFR em ter uma biblioteca possa ser seguido por outros hospitais. Assim, os hospitais poderão oferecer ao seu corpo clínico um espaço para o aprendizado constante, e, aos seus pacientes, médicos mais bem informados e capacitados para o tratamento das enfermidades no qual a informação de qualidade é essencial para melhorar a assistência em saúde. 


\section{AGRADECIMENTOS}

Meus agradecimentos a Professora Maria Ângela de Faria Resende, membro do Conselho Superior da Fundação Felice Rosso, pela revisão do português; a Dra. Marília de Abreu Martins de Paiva, Professora adjunta da ECl-UFMG pela revisão do conteúdo do artigo, a Renata Moscão Franco, Enfermeira da University of Arkansas for Medical Sciences (UAMS) pela correção do Abstract, e também ao Bibliotecário Gesner Francisco Xavier Junior, coordenador da Biblioteca J Baeta Viana da Faculdade de Medicina da UFMG, pela revisão final do artigo.

\section{REFERÊNCIAS}

ARCARI, R.; LAMB, G. The librarian in clinical care. The Hospital Medical Staff, v.6, n.12, p.1823,1977 .

BAHADORAN, Z. et al. The Principles of Biomedical Scientific Writing: Citation. International Journal of Endocrinology and Metabolism, Tehran, v.18, n.2, p.e102622, Apr 272020. doi: https://doi.org/10.5812/ijem.102622. Acesso em: 27 nov. 2019.

BERAQUET, V. S. M.; CIOL, R. Atuação do bibliotecário em ambientes não tradicionais: o campo da saúde. Pesquisa Brasileira em Ciência da Informação, João Pessoa, v.3, n.1, p.12737, jan./dez. 2010.

BERAQUET, V. S. M.; CIOL, R. O bibliotecário clínico no Brasil: reflexões sobre uma proposta de atuação em hospitais universitários. Datagrama Zero - Revista de Ciência da Informação, v.10, n.2, abr. 2009. Disponível em: http://www.datagramazero.org.br/abr09/Art 05.htm. Acesso em: 5 fev. 2019.

BRASIL. Lei no 4.084, de 30 de junho de 1962.Dispõe sobre a profissão de bibliotecário e regula seu exercício. Diário Oficial da União, Brasília, DF, 2 junho 1962. Disponível em: http://www.planalto.gov.br/ccivil 03/LEIS/1950-1969/L4084.htm. Acesso em: nov.27, 2019.

BRETTLE, A. et al. Evaluating clinical librarian services: a systematic review. Health Information and Libraries Journal, Oxford, v.28, n.1, p.3-22,2011.

CENTRO DE ESTUDOS DO HOSPITAL FELÍCIO ROCHO. Estatuto Social. Belo Horizonte: CEHFR, 2005. (Manuscrito).

CIOL, R.; BERAQUET, V. S. M. O. Evidência e informação: desafios da medicina para a próxima década. Perspectivas em Ciência da Informação, Belo Horizonte, v. 14, n. 3, p. 221-30, 2009. Disponível em: http://www.brapci.inf.br/v/a/7936. Acesso em: 27 nov. 2019.

CUNHA, M. B. Cooperação bibliotecária. Revista Ibero-americana de Ciência da Informação (RICl), Brasília, v. 11, n.2, p. 364-6, 2018. Disponível em: https://periodicos.unb.br/index.php/RICl/article/view/10932/9598. Acesso em: 27 nov. 2019. 
CUNHA, M. B. O papel do bibliotecário na sociedade brasileira. Revista da Escola de Biblioteconomia da UFMG, Belo Horizonte, v. 7, n. 1, 1978. Disponível em: http://hdl.handle.net/20.500.11959/brapci/75789. Acesso em: 27 nov. 2019.

DESCRITORES EM CIÊNCIAS DA SAÚDE: DeCS. 2020. ed. rev. e ampl. São Paulo: BIREME / OPAS / OMS, 2017. Disponível em: http://decs.bvsalud.org. Acesso em: 22 jul. 2020.

ESQUIFES. In: DICIO, Dicionário Online de Português. Porto: 7Graus, 2020. Disponível em: https://www.dicio.com.br/esquife/. Acesso em: 27 nov. 2019.

FERNANDES, M. R. Bibliotecário clínico: análise do perfil de um profissional dinâmico.2015. Dissertação (Mestrado em Ciência da Informação) - Escola de Ciência da Informação, Universidade Federal de Minas Gerais, Belo Horizonte, 2015.

FIORINI, N.; LIPMAN, D. J.; LU, Z. Cutting Edge: Towards PubMed 2.0.eLIFE, Cambridge, v.6, p.e28801, 2017.doi: https://doi.org/10.7554/eLife.28801. Acesso em: 27 nov. 2019.

FREITAS, L. V.; GOMES, H. F.; SANTOS, R. R. Ações mediadoras desenvolvidas pelos bibliotecários para estimular o uso dos livros eletrônicos. Tendências da Pesquisa Brasileira em Ciência da Informação, João Pessoa, v. 10, n. 1, p.1-19, 2017. Disponível em: https://revistas.ancib.org/index.php/tpbci/article/view/434/433. Acesso em: 01 nov. 2020.

GALVÃO, M. C. B. Do bibliotecário médico ao informacionista: traços semânticos de seus perfis e competências. Transinformação, Campinas, v. 20, n. 2, p. 181-91, ago. 2008. Disponível em: http://www.scielo.br/scielo.php?script=sci arttext\&pid=S010337862008000200006\&lng=en\&nrm=iso. Acesso em: 21 jul. 2020.

GERBER, A. L. The Librarian's Contribution to Continuing Medical Education. Medical Reference Services Quarterly, New York, v.36, n.4, p.408-14, Oct-Dec. 2017.

HAGEMAN, J. R. Medical Librarians Can Help Providers Improve Clinical Decision-Making and Education. Pediatric Annals, Thorofare, v.48, n.2, p.e49-e50, 2019.

HARRISON, J.; BERAQUET, V. Clinical librarians, a new tribe in the UK: roles and responsibilities. Health Information and Libraries Journal, Oxford, v.27, n.2, p.123-32, 2010. https://doi.org/10.1111/j.1471-1842.2009.00862.x.

HENDERSON, M. Data Management Roles for Librarians. In: HENDERSON, M. Data Management: A Practical Guide for Librarians. NY: Rowman \& Littlefield, 2016.

HILL, P. Report of a national review of NHS health library services in England: From knowledge to health in the 21st Century. 2008. Disponível em: http://citeseerx.ist.psu.edu/viewdoc/download?doi=10.1.1.462.9667\&rep=rep1\&type=pdf. Acesso em: 31 jan. 2019.

HOSPITAL FELICIO ROCHO. Quem Somos: missão. 2019. Disponível em: https://www.feliciorocho.org.br/. Acesso em: jan. 31, 2019. 
INSTITUTO BRASILEIRO DE INFORMAÇÃO EM CIÊNCIA E TECNOLOGIA (IBICT). Programa de Comutação Bibliográfica (COMUT): informações sobre o COMUT. Brasília, 2012. Disponível em: http://sitehistorico.ibict.br/informacao-para-ciencia-tecnologia-einovacao\%20/programa-de-comutacao-bibliografica-\%28comut\%29. Acesso em: 27 nov. 2019.

JESUS, D. L.; CUNHA, M. B. Produtos e serviços da web 2.0 no setor de referência das bibliotecas. Perspectivas em Ciência da Informação, Belo Horizonte, v. 17, n. 1, p. 110-33, 2012. Disponível em: http://hdl.handle.net/20.500.11959/brapci/38746. Acesso em: nov. 27, 2019.

LIPSCOMB, C. E. Clinical librarianship. Bulletin of the Medical Library Association, Chicago, v.88, n.4, p.393-5, 2000.

LOPES, A. A. Medicina Baseada em Evidências: a arte de aplicar o conhecimento científico na prática clínica. Revista da Associação Médica Brasileira, São Paulo, v. 46, n. 3, p. 285288, set. 2000. Disponível em http://www.scielo.br/scielo.php?script=sci arttext\&pid=S010442302000000300015\&lng=en\&nrm=iso. Acesso em: 22 out. 2020.

LYON, J. A. et al. The Lived Experience and Training Needs of Librarians Serving at the Clinical Point-of-Care. Medical Reference Services Quarterly, New York, v.34, n.3, p.311-33,2015.

NÚCLEO DE CIÊNCIAS DA SAÚDE FELÍCIO ROCHO. Regimento Interno do Núcleo de Ciências da Saúde Felício Rocho. Belo Horizonte: NCSFR, 2019. 9p. (Manuscrito).

PIRES, E. A. N.; RIBEIRO, C. P.; KLEBERSSON, M. Biblioteconomia clínica: espaço de atuação emergente para o bibliotecário no Século XXI. In: CONGRESSO BRASILEIRO DE BIBLIOTECONOMIA, DOCUMENTAÇÃO E CIÊNCIA DA INFORMAÇÃO, 25., 2013, Florianópois. Anais [...]Florianópolis: FEBAB, 2013. Disponível em: https://portal.febab.org.br/anais/article/view/1503. Acesso em: 27 nov. 2019.

PRAXIS SOFTWARES GERENCIAIS. Biblioteca Virtual do Centro de Estudos do Hospital Felício Rocho: i10bibliotecas versão 2020. Belo Horizonte: Praxis Softwares Gerenciais, 2020. Disponível em: https://centroestudoshfr.i10bibliotecas.com.br/. Acesso em: 21 jul. 2020.

RESENDE, M. Â. F; MARTINS, L. L. GOUVEIA, M. L. C. Memorial: Fundação Felice Rosso: 75 anos de Benemerência, 60 anos de Medicina. Belo Horizonte: Logos, 2012. 232p.

SACKETT, D. L. et al. Evidence-based medicine: what it is and what it isn't. British Medical Journal, London, v. 312, n.7023, p.71-2, 1996.

SANTOS, F. C. L.; MOLLICA, M. C. M.; GUEDES, V. L. S. Coerência na representação temática de artigos científicos na área de saúde pública. Perspectivas em Ciência da Informação, Belo Horizonte, v. 24, n. 3, p. 214-232, set. 2019. Disponível em:

http://www.scielo.br/scielo.php?script=sci arttext\&pid=S1413-

99362019000300214\&lng=en\&nrm=iso. Acesso em: 21 jul. 2020. 
SARACEVIC, T. Information Science. Journal of the American Society for Information Science, Silver Spring, v.50, n.12, p.1051-63, 1999.

SCHACHER, L. F. Clinical librarianship: its value in medical care. Annals of Internal Medicine, Philadelphia, v.134, n.8, p.717-20, 2001.

SHERA, J. S. The foundations of education for librarianship. New York: Wiley, 1972.

SILVA, F. C. C. A. Atuação do bibliotecário médico e sua interação com os profissionais da saúde para busca e seleção de informação especializada. Revista Digital de Biblioteconomia e Ciência da Informação, Campinas, v.3, n.1, p.131- 51, 2005. Disponível em:

https://periodicos.sbu.unicamp.br/ojs/index.php/rdbci/article/view/2057/2187. Acesso em: 27 nov. 2019.

SPENCER, A. J.; ELDREDGE, J. D. Roles for librarians in systematic reviews: a scoping review. The Journal of the Medical Library Association, Chicago, v. 106, n. 1, p. 46-56, jan. 2018.

VAN KESSEL, K. Gertrude Lamb's pioneering concept of the clinical medical librarian. Evidence Based Library and Information Practice, Alberta, v. 7, n. 1, p. 125-8, 2012.

VERGUEIRO, W. Desenvolvimento de coleções. São Paulo: Polis, APB, 1989. (Coleção Palavra Chave, 1). 96p.

WILLIS, S. R. NLM Classification 2019 Summer Edition Now Available. The NLM Technical Bulletin, Bethesda, n.430, e4, Sep-Oct 2019. Disponível em:

https://www.nlm.nih.gov/class/index.html. Acesso em: 21 jul. 2020.

WINNING, M. A, BEVERLEY, C. A. Clinical librarianship: a systematic review of the literature. Health Information and Libraries Journal, Oxford, v. 20, Suppl. 1, p. 10-21, 2003. 